\title{
Trauma Surgery $\&$ Acute Care Open \\ Health system factors associated with post-trauma mortality at the prehospital care level in Africa: a scoping review
}

\author{
Gilbert Koome (ㄱ), ${ }^{1}$ Martin Atela, ${ }^{2}$ Faith Thuita, ${ }^{1}$ Thaddaeus Egondi ${ }^{3}$
}

- Additional material is published online only. To view please visit the journal online (http://dx.doi.org/10.1136/ tsaco-2020-000530).

'School of Public Health University of Nairobi, Nairobi, Kenya

2Peterhouse, University of Cambridge, Cambridge, UK ${ }^{3}$ School of Mathematics, Strathmore University, Nairobi, Kenya

Correspondence to Gilbert Koome; rithaag@gmail. com

Received 13 June 2020 Accepted 30 July 2020

(C) Author(s) (or their employer(s)) 2020. Re-use permitted under CC BY-NC. No commercial re-use. See rights and permissions. Published by BMJ.

To cite: Koome G, Atela M Thuita F, et al. Trauma Surg Acute Care Open

2020;5:e000530.

\section{ABSTRACT}

Background Africa accounts forabout $90 \%$ of the global trauma burden. Mapping evidence on health systemfactors associated with post-trauma mortality is essential in definingpre-hospital care research priorities and mitigation of the burden. The studyaimed to map and synthesize existing evidence and research gaps on healthsystem factors associated with post-trauma mortality at the pre-hospital carelevel in Africa.

Methods A scoping review ofpublished studies and grey literature was conducted. The search strategyutilized electronic databases comprising of Medline, Google Scholar, Pub-Med,Hinari and Cochrane Library. Screening and extraction of eligible studies wasdone independently and in duplicate.

Results A total of 782 studytitles and or abstracts were screened. Of these, 32 underwent full text review. Out of the 32,17 met the inclusion criteria for final review. The majority of studies wereliterature reviews (24\%) and retrospective studies (23\%). Retrospective and qualitative studies comprised $6 \%$ of the includedstudies, systematic reviews $(6 \%)$, cross-sectional studies (17\%), Delphi studies $(6 \%)$, panel reviews $(6 \%)$ and qualitative studies (12\%), systematic reviews(6\%), cross-sectional studies (17\%), Delphi studies (6\%), panel reviews (6\%)and qualitative studies (12\%). Reported post-trauma mortality ranged from 13\%in Ghana to $40 \%$ in Nigeria. Reported preventable mortality is as high as $70 \%$ inSouth Africa, $60 \%$ in Ghana and $40 \%$ in Nigeria. Transport mode is the moststudied health system factor (reported in $76 \%$ of the papers). Only two studies $(12 \%)$ included access to pre-hospital care interventions aspects, nine studies $(53 \%)$ included care providers aspects and three studies (18\%) included aspectsof referral pathways. The types of transport mode and referral pathway are theonly factors significantly associated with post-trauma mortality, though thefindings were mixed. None of the included studies reported significantassociations between pre-hospital care interventions, care providers andpost-trauma mortality.

Discussion Although research onhealth system factors and its influence on post-trauma mortality at theprehospital care level in Africa are limited, anecdotal evidence suggests thataccess to pre-hospital care interventions, the level of provider skills andreferral pathways are important determinants of mortality outcomes. Thestrength of their influence will require well designed studies that couldincorporate mixed method approaches. Moreover, similar reviews incorporatingother LMICs are also warranted. Key Words: Health System Factors, EmergencyMedical Services [EMS], Pre-hospital Care, Post-Trauma mortality, Africa.

\section{BACKGROUND}

Globally, 5.8 million deaths (10\% of all deaths) are associated with traumatic injuries, ${ }^{1}$ of which road traffic crash accounts for over 1.2 million deaths and 50 million additional injuries. ${ }^{23}$ The majority (90\%) of the global trauma disease burden comprising traumatic deaths, preventable posttrauma, trauma mortality and morbidity occurs in low and middle-income countries (LMICs). ${ }^{45}$ Africa bears a disproportionate burden mainly due to poorly equipped health systems for management of the traumatic injuries both at the prehospital and the in-hospital care level. ${ }^{23}$

Prehospital care refers to care provided to trauma patients before or during transport to hospital by emergency medical service (EMS) providers or lay persons. A prehospital care system is an integral part of the EMS and the broader health systems that provides a systematic and organized immediate care to trauma patients at the trauma scene, during transport and throughout the out-of-hospital period. ${ }^{2}$ A key outcome measure of the prehospital care quality is the post-trauma mortality rates. In this study, the term post-trauma mortality (outcome variable of interest), refers to deaths occurring after trauma. On the other hand, prehospital care level refers to out-of-hospital settings where a casualty is attended to before successful evacuation and admission in a trauma care facility.

Health system elements such as transportation, healthcare professionals, life-saving interventions and referral pathways are critical in the provision of EMS and in preventing post-trauma mortality. Efficient and prompt prehospital evacuation or transport of trauma patients to a trauma care facility is an important determinant of post-trauma care outcome. ${ }^{6}$ This is because, prompt transportation of patients with proper care from the scene of injury to specialized trauma centers can significantly increase survival. ${ }^{7}$ It is estimated that $50 \%$ of the traumatic deaths occur due to evacuation delays at the prehospital care level. ${ }^{8}$ Preventable posttrauma mortality rate is significantly high in African countries compared with high-income countries. ${ }^{15}$

In many LMICs, more than half of trauma patients are transported in vehicles without proper handling capacity and skilled personnel to provide life-saving interventions. ${ }^{9}{ }^{10}$ Inadequate prehospital evacuation, limited skilled providers for critical trauma care and poor coordination and leadership $^{11-13}$ have left provision of prehospital care to unskilled lay responders and inadequately equipped 
and resourced private and non-governmental organizations to provide care to a large number of vulnerable populations. Notwithstanding, survival rates from the different modes of transport are not well documented and the existing evidence is too scanty and mixed to aid a reliable conclusion on their utility or benefits. ${ }^{214}$

Timely provision of quality care or life-saving interventions at the prehospital care level remains a key priority in improving post-trauma survival. Ultimately, the type of referral pathway and the choice of first referral facility (either direct or indirect to the trauma center). may play a fundamental role in reducing delays and improving survival as well as other prehospital care outcomes. ${ }^{715}$ Some studies recommend timely initiation of interventions at the injury scene and en route to hospital in line with the 'golden-hour' or the '60 minutes concept', which is a critical time period for successful life-saving or care intervention. ${ }^{16-18}$ The golden hour concept seeks to prevent avoidable timesensitive complications associated with irreversible pharmacological changes in the patients' body. ${ }^{17} 1920$ Other studies argue that access to professional prehospital care within 5 minutes of trauma and 8 minutes before advanced and specialized trauma care in a trauma facility or center is critical for survival and determination of the trauma outcome. ${ }^{21}$

At the injury scene in low-resource countries, trauma patients are less likely to receive any basic life support (BLS) care such as first aid and are thus transferred to the nearest hospital by untrained lay responders. ${ }^{12} 2223$ According to Boschini et al, ${ }^{22}$ direct transfer of trauma patients from the scene to the trauma center may confer a survival advantage by reducing time spent in referrals from peripheral hospitals. While this indirectly supports the value of the golden hour and ambulance transport in trauma distress responses, other studies refute this finding due to lack of strong evidence..$^{152124}$ In effect, the 'golden hour-60minute concept' remains a controversial subject in terms of care outcome benefits in LMICs.

There are two prehospital care interventions which can be provided at the prehospital care level by trained care providers: BLS and Advanced Life Support (ALS). ${ }^{6}$ In this study, we included lay responder, also known as 'No care' to refer to many of the patients who are not attended by a trained care provider at the prehospital care level. BLS involves providing adequate ventilation, oxygenation and securing of the airway. ${ }^{25}$ In most cases, prehospital deaths come as a result of uncontrolled hemorrhage, respiratory failure and airway obstruction which comprise BLS service package. As a result, provision of BLS interventions to trauma patients has been recommended extensively in the prehospital care set-up. ${ }^{26}$ Trained medical individuals, such as paramedics, emergency medical technicians, and trained bystanders, are expected to increase patient survival by providing this form of life-saving intervention. In addition to this level of care, developed countries have integrated use of ALS interventions in prehospital care but with limited adoption in LMICs such as Kenya. ALS is defined as a set of skills and life-saving protocols which extend BLS to further provide adequate breathing (ventilation), an open airway, and enable circulation such as rapid sequence induction, endotracheal intubation, cardiac defibrillation, cardiac monitoring, transcutaneous pacing, chest tube insertion, intravenous fluids and anesthesia induction, among others. Few patients receive BLS at the prehospital care level with most of trauma victims receiving no interventions due to lack of qualified medical practitioners and evacuation facilities. ${ }^{27}$

Nonetheless, evidence on the efficacy of ALS and BLS in prehospital trauma care remains controversial and scanty in
LMICs. ${ }^{628}$ At the global level, there is insufficient evidence and studies on efficacy, effectiveness and value of various prehospital care interventions in the prehospital settings. ${ }^{6}$ Moreover, the existing evidence is highly westernized with very few studies to allow for contextualization of similar evidence in LMICs. Even then, eliminating and/or reducing the trauma burden has increasingly gained momentum with advocacy support from WHO. ${ }^{1}$ Measures to reduce or eliminate trauma in LMICs such as adherence to road safety measures and traffic laws, use of personal protective equipment or gears like motorcycle helmets and inspection of the blood alcohol concentration for motorists seem to have yielded little in reducing mortality and disability. ${ }^{129}$ As a result, the WHO recommends that LMICs prioritize investment in local prehospital studies, scientific evidence documentation and defining locally adaptive health systems and policy reforms to augment the primary trauma and injury mitigation measures. ${ }^{12}$

This scoping review uses a health systems angle to identify and map the evidence on health system factors associated with posttrauma mortality at the prehospital care level. Documenting this evidence is key in defining and implementing resilient prehospital care systems for Africa and other low-resource countries.

\section{METHODOLOGY}

The review was based on the realist assumption that designing a resilient low-resource prehospital EMS system for traumatic injuries requires a health system-based approach in identifying fundamental components of the system and their influence on post-trauma mortality. Derived from inter-related and interdependent parts of a functional health system, ${ }^{31} 32$ the primary health system factors examined in this study comprise: type of prehospital care providers, prehospital transport mode, prehospital interventions provided and prehospital referral system or pathway used. While our focus was on health system factors associated with post-trauma mortality, the role of other contextual health system factors in the analysis framework was also considered using a systems approach. ${ }^{334}$ This includes financing, policy and legislative frameworks, health equipment and facilities like communication facilities and leadership.

The initial intention of this study was to complete a systematic review. However, a precursory research and subsequent review of the available evidence by the study team identified quality gaps that could not warrant a full systematic review. A scoping review was best suited for assembling an initial body of knowledge and identification of gaps to be addressed in future studies. The review was useful in obtaining a snapshot of available evidence to inform an appropriate study design in response to identified gaps in existing evidence..$^{35} 36$

A search was conducted for peer-reviewed articles and gray literature published in the English language between January 1, 2010 and December 31, 2019. The search was limited to studies that assessed health system factors associated with post-trauma mortality at the prehospital care level in Africa. No study design restrictions were applied to ensure a wider scope of evidence on the study subject. Both primary and secondary studies were included. The search strategy used a combination of the following key search terms: health system factors, factors, prehospital care, trauma mortality, survival and Africa. These were applied as follows: Search (Health System Factors OR Factors) AND (pre-hospital care) AND (Trauma Mortality OR Trauma Survival OR Trauma Functional Outcomes) . Searches were conducted in Medline, Google Scholar, PubMed, Hinari and Cochrane Library electronic databases and results imported into 


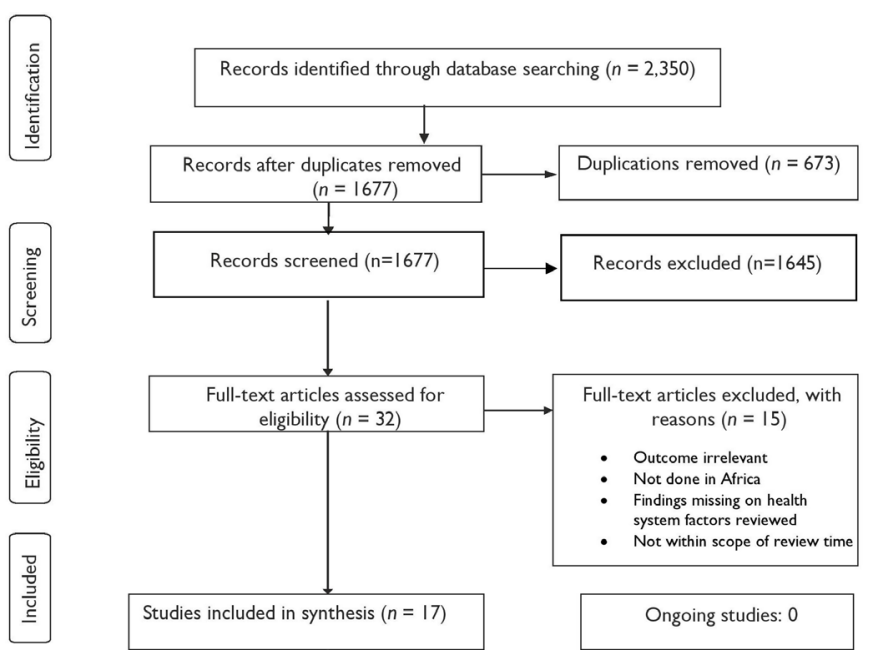

Figure 1 Flow diagram of study selection.

Mendeley, a reference management software. We also searched relevant study references and bibliographies.

Two reviewers independently reviewed study titles and abstracts for eligibility. Duplicate studies were identified and removed while any discrepancy was resolved by consensus and/ or involvement of a third-party adjustment. Retrieval of full text of studies meeting our study criteria was done for final eligibility review. This was done independently and in duplicate by two reviewers. Data extraction from the selected studies captured key data variables, namely author/s, year of publication, publication country, type of study (design) and key study findings based on our research aims articulated in the study background. A $10 \%$ sample of the eligible study data extraction was crosschecked for accuracy and completeness by a different reviewer. Extracted data were synthesized descriptively and using thematic analysis to map the existing body of literature in line with the objectives. Risk of bias was not performed since this was not a systematic review or meta-analysis. Extracted data were mapped and presented descriptively using frequencies and percentages and qualitative narratives.

\section{RESULTS}

The search generated 2350 studies. Duplicate studies (673) were excluded based on their titles whereas 750 were excluded on a review of their titles and abstracts. A total of 32 eligible articles underwent full-text review, out of which 15 studies were excluded as they did not meet the inclusion criteria. Seventeen studies met the inclusion criteria as shown in figure 1. Reasons for exclusion included wrong study locations, articles not published from year 2010 and later years, scope of findings such as lack of any relevant findings on study subject and non-research articles.

\section{Study characteristics}

Eight of the studies included in the synthesis were conducted between the years 2016 and 2019. Three of the studies were undertaken in South Africa whereas the rest werefrom across other countries in Africa. Four retrospective and four literature review articles were included in the study. Only two qualitative studies met our study inclusion criteria. There was no eligible prospective study or randomized clinical trials. Quantitative studies incorporated use of analytic techniques such as independent tests and multivariate analytic methods that adjust for possible confounders in the analysis were used as shown
Table 1 Description of studies reviewed

\begin{tabular}{|c|c|c|}
\hline Study characteristic & Frequency $(n=17)$ & Included studies (\%) \\
\hline \multicolumn{3}{|l|}{ Year } \\
\hline 2012 & 2 & 12 \\
\hline 2013 & 3 & 17 \\
\hline 2014 & 4 & 23 \\
\hline 2016 & 1 & 6 \\
\hline 2017 & 3 & 18 \\
\hline 2018 & 2 & 12 \\
\hline 2019 & 2 & 12 \\
\hline \multicolumn{3}{|l|}{ Countries of origin } \\
\hline Ethiopia & 1 & 6 \\
\hline Gabon & 1 & 6 \\
\hline Ghana & 2 & 12 \\
\hline Kenya & 1 & 6 \\
\hline Liberia & 1 & 6 \\
\hline Nigeria & 1 & 6 \\
\hline South Africa & 3 & 17 \\
\hline Tanzania & 2 & 12 \\
\hline Uganda & 2 & 12 \\
\hline Across Africa & 3 & 17 \\
\hline \multicolumn{3}{|l|}{ Study design } \\
\hline Retrospective & 4 & 23 \\
\hline Retrospective and qualitative study & 1 & 6 \\
\hline Systematic review & 1 & 6 \\
\hline Cross-sectional study & 3 & 17 \\
\hline Delphi study & 1 & 6 \\
\hline Literature review & 4 & 24 \\
\hline Multidisciplinary panel review & 1 & 6 \\
\hline Qualitative study & 2 & 12 \\
\hline
\end{tabular}

in table 1. None of the studies reviewed assessed the quality of evidence reported. There were no reviews (systematic, scoping or meta-analysis)on the study topic.

The primary outcome of interest was post-trauma mortality. Post-trauma mortality is reported to range from $13 \%$ in $\mathrm{Ghana}^{37}$ to $40 \%$ in Nigeria. ${ }^{5}$ Mortality rate was highest among brought-in dead, that is, prehospital care level, compared with in-hospital mortality, that is, at the trauma care facility level. ${ }^{124}{ }^{37}$ Reported preventable mortality was as high as $70 \%$ in South Africa, ${ }^{1} 60 \%$ in Ghana ${ }^{38}$ and $40 \%$ in Nigeria. ${ }^{5}$ In Nigeria and South Africa, delays to access specialized hospital care and insufficient intravenous fluid resuscitation en route to hospital were the main drivers of preventable mortality. ${ }^{15}$ In Nigeria, damage to the central nervous system, excessive bleeding (haemorrhage) and compromised airway leading to lack of adequate oxygen or difficulty in breathing (airway obstruction) were reported as the main causes of preventable post-trauma mortality. ${ }^{5}$ Online supplemental table 2 shows the summary findings on posttrauma mortality among the included studies. All the 17 studies focused on all forms of trauma. None of the studies narrowed the study scope (by design or analysis of disaggregation) to traumatic brain injuries (TBIs) despite its significant share of posttrauma mortality burden.

Health system factors associated with post-trauma mortality We examined the reported association between health system factors and post-trauma mortality at the prehospital care level. These factors comprised prehospital transport, interventions, 
providers and referral systems. Post-trauma transport mode and referral pathways were the only factors significantly associated with post-trauma mortality. Prehospital transport mode was reported by $76 \%$ of the reviewed articles. See online supplemental table 2 for a summary review finding on health system factors and their influence on post-trauma mortality.

\section{Prehospital transport mode}

From all factors reviewed prehospital transport was the most studied health system factor: 13 of the 17 studies included transport in their results as shown in online supplemental table 2. Except for South Africa, ${ }^{39}$ prehospital transport system for trauma victims in most countries included in the review studies is poorly developed. In most settings, there were inadequate ambulances to respond to trauma-related transport needs, and even in the few cases where ambulances were available, they were used as 'transport vehicles' rather than as 'emergency care vehicles'. ${ }^{2} 240$

In the reviewed studies, existing ambulances, and by extension, EMS response capacity of the respective countries were said to be ill equipped to provide quality life-saving interventions at injury scenes as well as en route care. ${ }^{11234142}$ In Morocco, out of 15 public ambulances assessed in the study site, only three were well equipped for emergency evacuation, with the rest only fitted with first aid equipment. ${ }^{8}$ In most countries of study, trauma patients were transported from injury scene using non-ambulatory means that included private and public vehicles. 243043 Private vehicle evacuation was the most common transport option. ${ }^{9102444}$ For example, in Ethiopia, only $20.3 \%$ of the sampled trauma patients arrived in the trauma management facilities through ambulance services, ${ }^{43}$ whereas in Kenya, less than $10 \%$ accessed ambulances. ${ }^{43}$ In South Africa, a country with one of the best prehospital EMS, $48.5 \%$ of patients used their own means of transport from the trauma scene to the nearest facility. ${ }^{39}$ Another South African study found patients with penetrating injuries to be often transported by non-EMS rather than EMS $(p<0.001)$. The study reported high mortality $(61 \%)$ among patients transported through EMS compared with nonEMS (38.9\%). ${ }^{24}$

Among the reviewed articles, prehospital time (time taken to evacuate a patient from the scene of injury to a specialized trauma facility after injury) is more than the recommended one 'golden hour'. ${ }^{40} 45$ In Ghana, the median transport time to a tertiary (specialized) trauma facility was 82 minutes, 22 more minutes than the recommended 60 minutes. ${ }^{37}$ No study studied differences in prehospital time in ambulance and non-ambulance transport means. While most of the reviewed studies reported transport means to be a significant predictor of post-trauma mortality, ${ }^{40} 45$ one South African study found no significant association between type of transport means and post-trauma mortality. ${ }^{24}$ Furthermore, the same study found prehospital transport time not to be a significant correlate of post-trauma mortality. ${ }^{24}$ In Malawi, transport by non-ambulance means such as private vehicles was also found to confer significant survival benefits as a result of prompt evacuation to a treatment facility and reduction of delays to life-saving interventions. ${ }^{22}$ None of the studies examined possible differences in post-trauma mortality based on ambulance ownership, public and private ambulance transport.

\section{Prehospital care or life-saving interventions}

Prehospital care interventions comprised use of BLS and ALS. Patients who do not receive any of the two interventions from a trained care provider like a paramedic, nurse or doctor at the prehospital care level are categorized to have received 'no care'. This is the least studied health system factor across the reviewed scope; none of the 17 articles reviewed examined the association between prehospital care interventions and post-trauma mortality. ${ }^{21} 23244546$ Only two studies, a Kenyan and a Tanzanian study, included prehospital care aspects in their results but did not examine their association with post-trauma mortality. ${ }^{40} 47$ One of the studies, a South African study, only compared use of intubations, an ALS intervention and in air versus ground emergency evacuations only. ${ }^{40}$ While the study investigated the success rate of intubations in the two modes of evacuation, there was no attempt to examine its effect on post-trauma mortality among the two modes of evacuation. The other study conducted in Ghana explored the potential for community-based trauma response through trained lay response care for trauma casualties. ${ }^{48}$ The study reported trained lay responders' care to be an effective first aid response but did not assess the effect of the lay responder rescue on post-trauma mortality. No findings on prehospital care outcomes were made across different prehospital care cadres like lay responders, paramedics and trained nurses.

\section{Prehospital care providers}

Nine of the 17 articles reviewed included different aspects of prehospital care providers, mainly descriptive and qualitative results on training and capacity levels. Shortage of skilled and qualified mainstream healthcare providers and, in particular, prehospital care providers to provide appropriate life-saving interventions is a key barrier to quality prehospital care. ${ }^{23} 2440$ Overall, lay responders such as the bystanders, policemen, friends, relatives and commercial drivers were the main providers of prehospital emergency rescue services from the injury scene to trauma care facilities. None of the studies examined the association between shortage and type of the providers on any posttrauma outcomes at the prehospital care level.

A study done in Liberia identified lay responder skills gap as a major competence challenge in providing critical rescue care at the prehospital care level. ${ }^{30}$ To respond to these gaps, countries such as Uganda and Ethiopia designed training programs targeting lay responders such as vehicle drivers and community volunteers aimed at equipping them with relevant first aid skills. The trainings were reported to improve quality of first aid interventions in Uganda and Ethiopia. ${ }^{23} 43$ In other counties like Tanzania, lay responder training has been suggested as necessity for strengthening capacity for community-based trauma responses. ${ }^{48}$ Two studies, one conducted by Edem et $a l$ in South Africa and another conducted by Sultan et al in Ethiopia, observed that current prehospital training offered to health professionals in the respective countries was inadequate in meeting the minimum skill and knowledge needs for critical trauma care. ${ }^{143}$ Furthermore, some of the studies reported that even though some countries such as Kenya had initiated EMS provider trainings, there was no success in improving prehospital care outcomes due to persistent skills gap among health professionals and facility capacity to provide quality care, especially in lower level facilities. ${ }^{1849}$ Interestingly, none of the reviewed studies documented evidence on the effects of different types of prehospital care providers on trauma mortality or the quality of life-saving interventions/care provided to the patients. Moreover, none of the studies examined whether there would be a difference in post-trauma outcomes if prehospital 
care interventions were provided by different cadres such as nurses, paramedics and lay responders at the prehospital care level.

\section{Prehospital referral pathways}

Only three studies included referral aspects in their findings. Two types of referral pathways were examined: direct and indirect referral pathways. Direct referral refers to direct transfer of patients from the injury scene to a specialized trauma care facility whereas indirect referral refers to transfer of casualties to a specialized trauma care facility through lower trauma facilities also known as peripheral facilities. Direct referral from the scene to a trauma care facility was the most common referral type compared with indirect referral at the prehospital care level. ${ }^{122} 24$ In Malawi, Boschini et al ${ }^{22}$ examined the relationship between referral pathways and post-trauma mortality and found direct referral from injury scene to a specialized trauma care facility to be significantly associated with post-trauma mortality $(p=0.006)$. Indirect transfer was found to be a mortality risk for severe trauma cases due to cases of delays and limited capacity to provide quality critical care in lower level facilities. ${ }^{18}$ The study found no statistically significant relationship between type of referral pathway and gender of the patient. Two studies, one in South African and another in Malawi, found that existing prehospital referral systems were weak with no supportive facilities and equipment such as ambulances and dispatch coordination centers which contributed to delays in care and avoidable mortality. ${ }^{130}$ In Liberia, pre-referral notification was found to be ineffective due to poorly organized and coordinated referral systems and lack of proper referral documentation and standardization of referral processes. ${ }^{30}$

\section{Other contextual factors}

Reviewed studies also identified policy context and modalities of prehospital emergency care communication and coordination to be important contextual health system factors which can potentially influence post-trauma care outcomes. In particular, inadequate prehospital policies and guidelines and/or lack of their implementation, and limited prehospital care capacity are reported as major challenges in Africa. ${ }^{523}$ In Nigeria, Adeloye and other authors found that there were no policies in relation to prehospital trauma care system. ${ }^{5}$ In Uganda, there was no national EMS policy or a functionalpostcrash care system across the health system. ${ }^{23}$ The study reported that inadequate or lack of health insurance cover and inability of victims of crash to raise ambulance fees were major challenges in provision of responsive EMS care at the prehospital care level..$^{23}$ Other barriers to optimal prehospital care outcomes identified among the reviewed studies included miscommunication about where to transfer patients, long patient waiting times in the prehospital settings, and limited intensive care unit ambulances which contributed to an increase in avoidable trauma deaths. ${ }^{11214950}$

\section{DISCUSSION}

Prehospital care in Africa is understudied. The quality of existing evidence on different aspects of health system factors and their influence on post-trauma mortality at the prehospital care level is low. In this study, mode of transport, referral systems, communication systems and post-trauma care and services are key aspects of the health systems reported in reviewed studies. Of these, transportation for trauma patients emerges as a major weak link and a key driver of poor post-trauma outcomes such as mortality and post-trauma complications. However, available evidence on transport modes and post-trauma mortality is both limited and mixed. Existing evidence has focused on ambulance and non-ambulance forms of transport. Currently, evidence on post-trauma survival benefits accrued from ambulance transport reflected is mixed. ${ }^{24} 4045$ In most cases, private vehicles (nonambulance transport) were cited to confer more survival benefits as opposed to ambulance transport. ${ }^{40} 45$ Studies affirm lack of well-coordinated and equipped ambulance transport across Africa. ${ }^{13} 51$ The studies acknowledged that vulnerable populations rely on private vehicles for emergency evacuation due to lack of readily available and well-equipped ambulance rescue services. This is partly due to low prioritization and investment in prehospital care (including research). Documentation on the role of ambulance ownership (public or private) on post-trauma mortality is lacking. In addition to being a study gap, provision of this evidence is fundamental in justifying a feasible financing model for ambulance evacuation that can contribute to reduction in post-trauma mortality.

Another important aspect of the mode of transport for prehospital trauma outcomes is the time it takes to move patients from trauma scene to a specialized trauma care hospital (prehospital time). Findings showed that patient waiting and evacuation time in the prehospital care settings was more than triple the international recommended response time of 8 minutes. ${ }^{52} 53$ Even though few studies included the effect of prehospital time on post-trauma mortality, the evidence suggests that delays at the prehospital care level are an important determinant of posttrauma mortality and morbidity. Most countries in Africa are reported to lack comprehensive trauma data systems or registries to capture evacuation time at different phases of care. This makes similar studies and analysis a challenge. Therefore, the effect of prehospital time on care outcomes is hardly investigated in literature. However, resourced communication and command center to support emergency rescue at the prehospital care level is equally weak and/or lacking in most countries. ${ }^{11} 21$ This communication facility is fundamental in supporting efficient prehospital evacuation and documenting vital evacuation data such as time for similar studies.

Direct referral of the trauma victims to a trauma management facility (without passing through lower or peripheral health facilities) has mixed results on post-trauma mortality. In some studies, indirect transfer through peripheral facilities has been argued to increase patient mortality ${ }^{22}$ whereas other studies report no association with mortality. ${ }^{21}{ }^{24}$ The current evidence is very limited to support a validated conclusion on this association; in our review, only three studies reported on this outcome. It has been established that existing referral systems are not effective and not well linked to trauma facilities, a possible explainer for higher mortality in indirect referral options. ${ }^{52} 53$ Furthermore, existing prehospital referral systems are poorly organized and lack effective linkages with specialized trauma care facilities and communication/command centers for prompt responses and continuity of care which in turn causes avoidable evacuation delays at prehospital care level. ${ }^{30} 54$ Referral documentation and coordination is equally poor; many patients are transferred to the next available health facility irrespective of their capacity to provide required care. ${ }^{30}$ Many of these facilities are not equipped to provide the indicative critical care and life-saving interventions. ${ }^{3055}$ This affects the continuity of prehospital care interventions, hence increasing risk of avoidable mortality.

A third health system feature linked to effective referral and post-trauma outcomes is emergency rescue communication or command centers connecting all trauma care facilities (both lower level and specialized facilities) with prehospital 
care level. Because of an almost non-existent EMS policy and communication guidelines/systems at prehospital care level to support trauma responses, many patients are forced to depend on existing informal prehospital care and referral systems which may increase trauma mortality and poor outcomes risks. ${ }^{30}$ This is affirmed by the high proportion of patients transported from injury scene by lay responders with no life-saving skills ${ }^{4856-58}$ and the overwhelming evidence of limited capacity of lower level facilities to provide basic life-saving interventions in case of indirect referrals. ${ }^{30415960}$ In many instances, these patients are transported to facilities without capacity to provide required care which puts patients' survival at risk. The weak and vulnerable health system increases risk to poor post-trauma outcomes like avoidable deaths, disability and other health complications. No evidence was found on the role of informed referral decisions (access to reliable information on availability of necessary facilities, resources or equipment to support appropriate referral pathways such as to facility with required treatment capacity) on post-trauma mortality at the prehospital care level. A key gap established was the limited mapped evidence on referral systems which did not differentiate the role of on-scene referrals by different types of providers and availability of information on capacity of the facility to manage the trauma. Yet, understanding these referral patterns and pathways can provide insight and knowledge on referral pathway practices at the prehospital care level while illuminating opportunities for possible mitigation measures to reduce mortality as a result of delays and suboptimal referral pathway decisions.

Prehospital care interventions for trauma victims through EMS are another aspect of the health system reported. The reviewed studies were in concurrence with global literature which identify poor EMS at the prehospital care level as a major drawback in reducing high and increasing trauma burden, especially in lowresource countries. ${ }^{1152122243055}$ Inability to access quality services is attributed to the perceived weak health systems and response capacity at prehospital care level. A particular aspect of the weakness in the health system at the prehospital level is delays to life-saving interventions, also linked to high risk of preventable mortalities. ${ }^{11134}$ Despite limited evidence on this health system factor, provision of timely and quality life-saving interventions at the scene of injury and en route to a trauma care facility has been linked to improved health outcomes. ${ }^{58}$ On the other hand, fragmented, disjointed and poorly resourced EMS systems continue to create major challenges to post-trauma survival. ${ }^{15} 55$ Our evidence fell short of defining efficacy of different types of life-saving interventions provided in Africa. While life-saving interventions are the core effective trauma responses, evidence associating the interventions such as BLS and ALS with posttrauma mortality is grossly lacking in African scientific literature. This confirms the limited investment and prioritization of health system research at prehospital care level. This evidence is critical in defining minimum life-saving intervention packages for an effective and responsive local trauma system and responses. Research questions on efficacy of specific interventions at the prehospital care level are important for low-resource countries in Africa seeking to develop robust EMS systems at the prehospital care level.

In addition to prehospital delays, barrier to quality care and outcomes was also attributed to inadequate or, in some cases, lack of trained and skilled personnel such as paramedics to attend to the injured victims on-site. Consequently, untrained lay responders such as family members, good Samaritans or policemen, with limited or no critical care skill/expertise, end up offering the needed rescue services. The situation is complicated by limited communication facilities and equipment to support and enable facility-based practitioners to swiftly respond to trauma distress calls at the prehospital care level while complimenting lay responder rescue assistance. ${ }^{61-63}$ Due to skill and rescue capacity gaps, lay responder rescue and interventions could be contributing to patient mortality and post-trauma complications. Despite lack of evidence to confirm or refute the assumption, lack of critical care skills could heighten risk of avoidable damages and complications.

Efficient and effective prehospital trauma system combines minimum transport time with adequate resuscitation provided by a qualified and skilled care provider. ${ }^{64} 858$ This is often not the case with untrained lay responder rescues due to lack of essential critical care competence. Training lay responders on basic first aid can play a significant role in improving posttrauma survival in low-resource countries or environments. ${ }^{15657}$ Training lay responders can also be useful in addressing trained provider quality and quantity (capacity) gaps in low-resource environments. ${ }^{15} 5657$ Hiring, training, equipping and sustaining both quality prehospital care professionals and life-saving interventions have significant resource implications. In addition to other health professionals/cadres, identifying and documenting evidence on value-add for both untrained and trained lay responders on post-trauma mortality remains an important research objective for future prehospital care studies conducted in Africa. With respect to methods assessment, there is no strong evidence on quality of prehospital care evidence in Africa. Our review affirms gaps in types of study methods used. Most studies are retrospective and literature reviews. There is limited diversity in study types and methods which negatively affects the quality of literature evidence available for policy and programmatic interventions. For instance, prospective studies are lacking whole triangulation of data sources and methodology is limited. This reflects the lack of organized trauma data and registries collecting trauma-related data to support relevant studies at this level of care. ${ }^{266566}$ In most African countries, trauma registry or data on health system factors such as type of care providers, prehospital response times and type of intervention are not routinely collected and documented. Prehospital care data are fragmented in both manual and electronic databases ${ }^{8}$ while in most cases, it is not collected and/or collated. Adopting complementariness in various data sources held in both prehospital and in-hospital care levels can enrich quality of data. Even though the process of collating the data can be both laborious and expensive, computerization of the prehospital care and information systems and integrating it with other mainstream health systems at in-hospital care level may address this challenge and improve availability of actionable evidence at prehospital care level.

\section{Limitations}

Our review was limited to African settings. Our initial intention was to conduct a systematic review. However, a cursory search and subsequent team review of the available evidence identified quality gaps that limited the ability to perform full systematic review. We settled on a scoping review with an aim of obtaining a snapshot of available evidence to inform appropriate study priorities and designs in response to identified evidence gaps.$^{35} 36$ Consequently, many of the included studies did not report or include statistical association models and findings on the factors investigated further limiting the level of evidence deduced from the articles. Health system factors incorporate wide range of study scope. The search strategy terms, which combined aspects of health system factors, post-trauma mortality, prehospital 
care and study settings, captured a wide range and number of screening studies most of which were not adequately relevant to the study variables. The studies were found to include limited evidence on variables of interest though useful in mapping and identifying possible prehospital care research gaps and priorities to guide future studies.

\section{CONCLUSION}

Africa is one of the continents with a high share of preventable post-trauma morbidity and mortality yet its EMS system is poorly developed and ill equipped to adequately respond to trauma distress. Preventable post-trauma mortality is highest at the prehospital care level. However, prehospital care is understudied. There is a huge health system research gap (evidence) to support the development of locally responsive emergency trauma care responses at this care level. Except for prehospital care transport mode, evidence on other health system factors and their influence on post-trauma mortality at the prehospital care level is either unavailable and/or limited. No evidence was found to confirm or refute the possibility of a significant relationship between types of prehospital care providers, prehospital care interventions and post-trauma mortality. These study gaps are due to limitation in the number of local studies, quality and diversity of methods applied. Our findings highlight a need for increase in local health systems research at the prehospital care level, in particular, for TBIs. Increasing prospective, qualitative and mixed methods studies is indicated to enhance the quality and level of local evidence to support informed prehospital care investment. Similar reviews incorporating other LMICs are also warranted.

Acknowledgements The authors would like to thank Dr Leila Abdullahi Hussein, Mr Boniface Muthomi, Ms Winfred Kananu and Mr Martin Mwenda for their technical assistance in this review.

Contributors The research reported in this paper emanates from a larger PhD study on health system factors associated with pre-hospital care outcomes among traumatic patients in Kenya. GK is study PI, MA is the primary supervisor. FT and TE are secondary supervisors. GK and MA conceptualized the study, conducted the literature search, data analysis and wrote the manuscript. FT and TE reviewed the manuscript. All contributors read and approved the final article.

Funding The study was undertaken as part of a PhD program with full tuition scholarship from the Higher Education Loans Board (HELB) of Kenya.

Competing interests None declared.

Patient consent for publication Not required.

Ethics approval This study was granted ethical approval and consent waiver by the Kenyatta National Hospital and University of Nairobi (KNH-UoN) Ethics and Research Committee (ERC) reference number KNH-UoN/ERC/FORM/IC05.

Provenance and peer review Not commissioned; externally peer reviewed.

Open access This is an open access article distributed in accordance with the Creative Commons Attribution Non Commercial (CC BY-NC 4.0) license, which permits others to distribute, remix, adapt, build upon this work non-commercially, and license their derivative works on different terms, provided the original work is properly cited, appropriate credit is given, any changes made indicated, and the use is non-commercial. See: http://creativecommons.org/licenses/by-nc/4.0/.

\section{ORCID iD}

Gilbert Koome http://orcid.org/0000-0003-4702-5232

\section{REFERENCES}

1 Edem IJ, Dare AJ, Byass P, D'Ambruoso L, Kahn K, Leather AJM, Tollman S, Whitaker J, Davies J. External injuries, trauma and avoidable deaths in Agincourt, South Africa: a retrospective observational and qualitative study. BMJ Open 2019;9:e27576-11.

2 WHO. Regional action plan for violence and injury prevention in the Western Pacific (2016-2020), 2016

3 World Health Organization. Injuries and violence: the facts. 2014. http://apps. who.int/ iris/bitstream/10665/149798/1/9789241508018_eng.pdf?ua=1\&ua=1\&ua=1.
4 Obermeyer Z, Abujaber S, Makar M, Stoll S, Kayden SR, Wallis LA, Reynolds TA. Emergency care in 59 low- and middle-income countries: a systematic review. Bull World Health Organ 2015;93:577-86.

5 Adeloye D. Prehospital trauma care systems: potential role toward reducing morbidities and mortalities from road traffic injuries in Nigeria. Prehosp Disaster Med 2012:27:536-42.

6 Sanghavi P, Jena AB, Newhouse JP, Zaslavsky AM. Outcomes of basic versus advanced life support for out-of-hospital medical emergencies. Ann Intern Med 2015;163:681-90.

7 Georgiou A, Lockey DJ. The performance and assessment of hospital trauma teams. Scand I Trauma Resusc Emerg Med 2010;18:66-7.

8 Plummer V, Boyle M. Ems systems in lower-middle income countries: a literature review. Prehosp Disaster Med 2017:32:64-70.

9 Zafar SN, Haider AH, Stevens KA, Ray-Mazumder N, Kisat MT, Schneider EB, Chi A, Galvagno SM, Cornwell EE, Efron DT, et al. Increased mortality associated with EMS transport of gunshot wound victims when compared to private vehicle transport. Injury 2014;45:1320-6.

10 McCoy CE, Menchine M, Sampson S, Anderson C, Kahn C. Emergency medical services out-of-hospital scene and transport times and their association with mortality in trauma patients presenting to an urban level I trauma center. Ann Emerg Med 2013;61:167-74

11 Reynolds TA, Mfinanga JA, Sawe HR, Runyon MS, Mwafongo V. Emergency care capacity in Africa: a clinical and educational initiative in Tanzania. J Public Health Policy 2012;33:126-37.

12 Mehmood A, Rowther AA, Kobusingye O, Hyder AA. Assessment of pre-hospital emergency medical services in low-income settings using a health systems approach. Int J Emerg Med 2018;11:53.

13 Raj R, Siironen J, Kivisaari R, Kuisma M, Brinck T, Lappalainen J, Skrifvars MB. Factors correlating with delayed trauma center admission following traumatic brain injury. Scand I Trauma Resusc Emerg Med 2013;21:67.

14 Suriyawongpaisal P, Aekplakorn W, Tansirisithikul R. A Thailand case study based on quantitative assessment: does a national lead agency make a difference in prehospital care development in middle income countries? Scand I Trauma Resusc Emerg Med 2014;22:75.

15 Balikuddembe JK, Ardalan A, Khorasani-Zavareh D, Nejati A, Raza O. Weaknesses and capacities affecting the prehospital emergency care for victims of road traffic incidents in the greater Kampala metropolitan area: a cross-sectional study. BMC Emerg Med 2017;17:29.

16 Curtis K, McCarthy A, Mitchell R, Black D, Foster K, Jan S, Burns B, Tall G, Rigby O, Gruen $R$, et al. Paediatric trauma systems and their impact on the health outcomes of severely injured children: protocol for a mixed methods cohort study. Scand I Trauma Resusc Emerg Med 2016;24:1-8.

17 Lerner EB, Moscati RM. The golden hour: scientific fact or medical "urban legend"? Acad Emerg Med 2001;8:758-60.

18 Pham H, Puckett Y, Dissanaike S. Faster on-scene times associated with decreased mortality in helicopter emergency medical services (HemS) transported trauma patients. Trauma Surg Acute Care Open 2017;2:e000122.

19 Lyon RM, Perkins ZB, Chatterjee D, Lockey DJ, Russell MQ. Significant modification of traditional rapid sequence induction improves safety and effectiveness of pre-hospital trauma anaesthesia. Crit Care 2015:19:134.

20 Newgard CD, Schmicker RH, Hedges JR, Trickett JP, Davis DP, Bulger EM, Aufderheide TP, Minei JP, Hata JS, Gubler KD, et al. Emergency medical services intervals and survival in trauma: assessment of the "golden hour" in a North American prospective cohort. Ann Emerg Med 2010;55:235-46.

21 Williams T, Finn J, Fatovich D, Jacobs I. Outcomes of different health care contexts for direct transport to a trauma center versus initial secondary center care: a systematic review and meta-analysis. Prehosp Emerg Care 2013;17:442-57.

22 Boschini LP, Lu-Myers Y, Msiska N, Cairns B, Charles AG. Effect of direct and indirect transfer status on trauma mortality in sub Saharan Africa. Injury 2016;47:1118-22.

23 Balikuddembe JK, Ardalan A, Khorasani-Zavareh D, Nejati A, Kasiima S. Factors affecting the exposure, vulnerability and emergency medical service capacity for victims of road traffic incidents in Kampala metropolitan area: a Delphi study. BMC Emerg Med 2017;17:1-8.

24 Möller A, Hunter L, Kurland L, Lahri Sa'ad, van Hoving DJ, Lahri S, ad L. The association between hospital arrival time, transport method, prehospital time intervals, and inhospital mortality in trauma patients presenting to Khayelitsha Hospital, Cape town. Afr J Emerg Med 2018;8:89-94.

25 Thompson L, Hill M, Davies C, Shaw G, Kiernan MD. Identifying pre-hospital factors associated with outcome for major trauma patients in a regional trauma network: an exploratory study. Scand I Trauma Resusc Emerg Med 2017:25:83.

26 Taibo CLA, Moon TD, Joaquim OA, Machado CR, Merchant A, McQueen K, Sidat $M$, Folgosa E. Analysis of trauma admission data at an urban hospital in Maputo, Mozambique. Int J Emerg Med 2016;9:1-7.

27 Gathecha GK, Githinji WM, Maina AK. Demographic profile and pattern of fata injuries in Nairobi, Kenya, January-June 2014. BMC Public Health 2017;17:34.

28 Rognås L, Hansen TM, Kirkegaard H, Tønnesen E. Pre-hospital critical care anaesthesiologists and traumatic brain injury-guideline adherence. Scand I Trauma Resusc Emerg Med 2014;22 
29 Strnad M, Lesjak VB, Vujanović V, Pelcl T, Križmarić M. Predictors of mortality and prehospital monitoring limitations in blunt trauma patients. Biomed Res Int 2015;2015:1-7.

30 Kim J, Barreix M, Babcock C, Bills CB. Acute care referral systems in Liberia: transfer and referral capabilities in a low-income country. Prehosp Disaster Med 2017;32:642-50.

31 Who-Glossary. Health systems strengthening: glossary. 2012. http://www.who.int/ healthsystems/hss_glossary/en/index.html.

32 WHO. Monitoring the building blocks of health systems: a handbook of indicators and their measurement strategies. 2010. http://www.who.int/healthinfo/systems/WHO_ MBHSS_2010_full_web.pdf.

33 Von BL. General system theory. 1976. https://www.amazon.com/General-SystemTheory-Author-Bertalanffy/dp/B00Y30TOHS (11 Mar 2020).

34 Dawson B. Bertalanffy revisited: Operationalizing a general systems theory based business model through General systems theory thinking, modeling, and practice. Int Soc Syst Sci 2007:1201-13.

35 Peterson J, Pearce PF, Ferguson LA, Langford CA. Understanding scoping reviews: definition, purpose, and process. J Am Assoc Nurse Pract 2017;29:12-16.

36 Lockwood C, Dos Santos KB, Pap R. Practical guidance for knowledge synthesis: Scoping review methods. Asian Nurs Res 2019;13:287-94.

37 Mahama M-N, Kenu E, Bandoh DA, Zakariah AN. Emergency response time and pre-hospital trauma survival rate of the National ambulance service, greater Accra (January - December 2014). BMC Emerg Med 2018;18:3-9.

38 Yeboah D, Mock C, Karikari P, Agyei-Baffour P, Donkor P, Ebel B. Minimizing preventable trauma deaths in a limited-resource setting: a test-case of a multidisciplinary panel review approach at the Komfo Anokye teaching hospital in Ghana. World J Surg 2014;38:1707-12.

39 Gunning M, Perkins Z, Crilly J, von Rahden R. Paramedic rapid sequence intubation (RSI) in a South African emergency medical service (EMS) is effective, but is it safe? Scand I Trauma Resusc Emerg Med 2013;21:S29.

40 Botchey IM, Paruk F, Wako D, Gachari W, Kibias S, Hyder AA, Stevens KA. 785 Development of a national EMS policy for Kenya: opportunities for action. Inj Prev 2016:22:A281.1-A281.

41 Coyle RM, Harrison H-L. Emergency care capacity in Freetown, Sierra Leone: a service evaluation. BMC Emerg Med 2015;15:2.

42 Murthy S, Leligdowicz A, Adhikari NKJ. Intensive care unit capacity in low-income countries: a systematic review. PLoS One 2015;10:e0116949.

43 Sultan M, Abebe Y, Tsadik AW, Ababa A, Yesus AG, Mould-Millman N-K. Trends and barriers of emergency medical service use in Addis Ababa; Ethiopia. BMC Emerg Med 2019;19:28.

44 Demetriades D, Chan L, Cornwell E, Belzberg H, Berne TV, Asensio J, Chan D, Eckstein M, Alo K. Paramedic vs private transportation of trauma patients. Effect on outcome. Arch Surg 1996;131:133-8.

45 Chalya PL, Mabula JB, Dass RM, Mbelenge N, Ngayomela IH, Chandika AB, Gilyoma JM. Injury characteristics and outcome of road traffic crash victims at Bugando medical centre in northwestern Tanzania. J Trauma Manag Outcomes 2012;6:1-8.

46 Tiska MA, Adu-Ampofo M, Boakye G, Tuuli L, Mock CN. A model of prehospital trauma training for lay persons devised in Africa. Emerg Med J 2004:21:237-9.

47 Kuzma K, Lim AG, Kepha B, Nalitolela NE, Reynolds TA, Georg LA, Evelyn. RTA. The Tanzanian trauma patients' prehospital experience: a qualitative interview-based study. BMJ Open 2015;5:e006921.
48 Kuzma K, Lim AG, Kepha B, Nalitolela NE, Reynolds TA, Georg LA, E velyn NN. The Tanzanian trauma patients' prehospital experience: a qualitative interview-based study. BMJ Open 2015;5:e006921.

49 Adeloye D, Thompson JY, Akanbi MA, Azuh D, Samuel V, Omoregbe N, Ayo CK. The burden of road traffic crashes, injuries and deaths in Africa: a systematic review and meta-analysis. Bull World Health Organ 2016;94:510-21.

50 Bosson N, Redlener MA, Foltin GL, Raven MC, Foran MP, Wall SP. Barriers to utilization of pre-hospital emergency medical services among residents in Libreville, Gabon: a qualitative study. African J Emerg Med 2013;3:172-7.

51 Roy N, Kizhakke Veetil D, Khajanchi MU, Kumar V, Solomon H, Kamble J, Basak D, Tomson G, von Schreeb J. Learning from 2523 trauma deaths in India- opportunities to prevent in-hospital deaths. BMC Health Serv Res 2017;17:142.

52 Bigdeli M, Khorasani-Zavareh D, Mohammadi R. Pre-hospital care time intervals among victims of road traffic injuries in Iran. A cross-sectional study. BMC Public Health 2010;10:406.

53 Henry JA, Reingold AL. Prehospital trauma systems reduce mortality in developing countries. J Trauma Acute Care Surg 2012;73:261-8.

54 Bundu I, Lowsby R, Vandy HP, Kamara SP, Jalloh AM, Scott COS, Beynon F. The burden of trauma presenting to the government referral hospital in Freetown, Sierra Leone: an observational study. Afr J Emerg Med 2019;9:\$9-13.

55 Haghparast-Bidgoli $H$, Hasselberg M, Khankeh $H$, Khorasani-Zavareh D, Johansson E. Barriers and facilitators to provide effective pre-hospital trauma care for road traffic injury victims in Iran: a grounded theory approach. BMC Emerg Med 2010;10:10-20.

56 Jayaraman S, Mabweijano JR, Lipnick MS, Caldwell N, Miyamoto J, Wangoda R, Mijumbi C, Hsia R, Dicker R, Ozgediz D, et al. First things first: effectiveness and scalability of a basic prehospital trauma care program for lay first-responders in Kampala, Uganda. PLoS One 2009:4:e6955-7.

57 Heidari M, Aryankhesal A, Khorasani-Zavareh D. Laypeople roles at road traffic crash scenes: a systematic review. Int J Inj Contr Saf Promot 2019;26:82-91.

58 Murad MK, Larsen S, Husum H. Prehospital trauma care reduces mortality. ten-year results from a time-cohort and trauma audit study in Iraq. Scand J Trauma Resusc Emerg Med 2012;20:13.

59 Sasser S, Varghese M. Prehospital trauma care Sytems, 2005.

60 Dharap SB, Kamath S, Kumar V. Does prehospital time affect survival of major trauma patients where there is no prehospital care? J Postgrad Med 2017;63:169.

61 Harmsen AMK, Geeraedts LMG, Giannakopoulos GF, Terra M, Christiaans HMT, Mokkink LB, Bloemers FW. National consensus on communication in prehospital trauma care, the DENIM study. Scand I Trauma Resusc Emerg Med 2017:25:67.

62 O'Hara R, Johnson M, Siriwardena AN, Weyman A, Turner J, Shaw D, Mortimer P, Newman C, Hirst E, Storey $M$, et al. A qualitative study of systemic influences on paramedic decision making: care transitions and patient safety. J Health Serv Res Policy 2015;20:45-53.

63 Pinet LM. [Prehospital emergency care in Mexico City: the opportunities of the healthcare system]. Salud Publica Mex 2005;47:64-71.

64 Usselman C. HHs public access. Physiol Behav 2017:176:139-48.

65 Tansley G, Schuurman N, Amram O, Yanchar N. Spatial access to emergency services in low- and middle-income countries: a GIS-based analysis. PLoS One 2015; 10:e0141113-12

66 Mould-Millman N-K, Sasser SM, Wallis LA. Prehospital research in sub-Saharan Africa: establishing research tenets. Acad Emerg Med 2013;20:1304-9. 